\title{
El nuevo sistema de acreditación para el acceso a los cuerpos de profesorado universitario
}

\author{
The new system of accreditation for acces to \\ the university teaching staff
}

\author{
Ramón Galindo Caldés \\ Doctor en Derecho. Profesor Asociado \\ de Derecho Administrativo. Universitat de \\ Barcelona (UB). \\ Email: rlgalindo@ub.edu

\section{Llorenç Arguimbau Vivó} \\ Doctor en Documentación. Profesor Colaborador \\ Universitat Oberta de Catalunya (UOC). \\ Email: larguimbau@uoc.edu
}

\section{Resumen}

La selección para el acceso a los cuerpos docentes universitarios se basa, desde 2007, en el sistema de acreditación, que implica que se reconoce a los candidatos un umbral mínimo de calidad para el acceso a plazas de profesorado funcionario. El proceso está basado en la evaluación de los méritos de los candidatos, especialmente en docencia e investigación, para garantizar que cumplen con unos requisitos mínimos. Después de casi diez años de vigencia, el sistema de acreditación se modifica con cambios importantes. Este trabajo analiza los aspectos más relevantes de este cambio de sistema, especialmente en lo que se refiere a la organización de las comisiones de acreditación, el procedimiento $\mathrm{y}$, por último, la baremación de los méritos de los candidatos a obtener la acreditación.

\section{Palabras clave}

Evaluación, Universidad, Acreditación, Personal docente, ANECA 


\section{Abstract}

Selection for access to university teaching is based, since 2007, in the accreditation system, which means that a minimum quality threshold is recognized for access to faculty positions. The process is based on the assessment of the merits of the candidates, especially in teaching and research, to ensure that they meet minimum requirements. After almost ten years of existence, the accreditation system is reformed with important changes. This paper analyses the most relevant aspects of this reform, especially regarding the organization of accreditation committees, the procedure and, finally, the merit standards of the candidates to obtain accreditation.

\section{Key words}

Evaluation, University, Accreditation, University teaching staff, ANECA. 


\section{Introducción}

La endogamia universitaria ha sido un tema recurrente en lo que se refiere a la provisión de plazas para profesorado universitario ${ }^{1}$, y ha estado, en parte, en el origen de la introducción de un sistema de selección del profesorado para el acceso a los cuerpos docentes, que se produce a través de la Ley Orgánica 6/2001, de 21 de diciembre, de Universidades (LOU). La LOU introduce la habilitación nacional como sistema de evaluación. A grandes rasgos ${ }^{2}$, estaba basado en una prueba presencial de los candidatos a la habilitación ante una comisión de siete miembros, todos ellos profesores Titulares de Universidad (TU) (con al menos un sexenio de investigación) o Catedráticos de Universidad (CU) (con al menos dos sexenios de investigación). El sistema recibió numerosas críticas por su coste, no eliminación de la endogamia, etc. (BASTIDA FREIJEDO, $2015,11)$, y fue sustituido pocos años más tarde por el de acreditación.

El sistema de acreditación del profesorado se crea con la Ley Orgánica 4/2007, de 12 de abril, por la que se modifica la Ley Orgánica 6/2001, de 21 de diciembre, de Universidades (LOMLOU). El nuevo modelo, en sustitución del de habilitación, se establece para el acceso a dos tipos de cuerpos de profesorado universitario funcionario, que el nuevo art. 56.1 LOU diferencia entre el de CU y el de Profesores TU. Su intención es, tal y como se expone en su preámbulo, permitir que las universidades seleccionen a su profesorado entre candidatos previamente acreditados. Se afirma que el nuevo sistema incorpora para el conjunto de la comunidad académica un mayor rigor en la acreditación y una mayor flexibilidad para las universidades en la selección de su personal.

El sistema de acreditación se desarrolla a través del Real Decreto 1312/2007, de 5 de octubre, por el que se establece la acreditación nacional para el acceso a los cuerpos docentes universitarios, y sustituye las pruebas del modelo de habilitación por una presentación de méritos, que son valorados por una comisión. No se condicionan por tanto el número de acreditados a las plazas disponibles, sino que se separan las fases de acreditación y concurso, de forma que la primera simplemente certifica que el candidato cumple con unos estándares mínimos que le permiten optar a una plaza. De esta forma, como ya se ha señalado, se produce un desplazamiento del carácter competitivo del sistema de selección a los concursos de acceso a las plazas (ALEGRE ÁVILA, 2007, 446).

El sistema de acreditación no ha eliminado las críticas, aunque se ha consolidado en los últimos diez años. Con la aprobación del Real Decreto 415/2015, de 29 de mayo, de modificación del Real Decreto 1312/2007, de 5 de octubre, se producen cambios relevantes en la estructuración del sistema, que se analizarán a lo largo de este trabajo. Los objetivos que pretende el RD 415/2015 son la simplificación normativa, la mejora regulatoria de los procedimientos de acreditación, y garantizar mayor objetividad y transparencia en la acreditación, entre otros. En este trabajo abordaremos tres aspectos del nuevo sistema, como son su organización -especialmente a través de las comisiones evaluadoras-; algunos elementos sobre el proceso de evaluación -

1Vid., entre otros, BASTIDA FREIJEDO, 2015; ALEGRE ÁVILA, 2007; DEL SAZ CORDERO, 1997; NIETO, 1984.

2 El sistema de habilitación nacional fue desarrollado por el Real Decreto 774/2002, de 26 de julio. 
especialmente la eliminación de los informes externos-; y, por último, los criterios de evaluación.

El nuevo sistema ha tenido una entrada en vigor atípica, en cuanto a que ésta se supeditó a la aprobación del Estatuto de la Agencia Nacional de Evaluación de la Calidad y Acreditación (ANECA). Así, en la Disposición Final 4a del RD 415/2015 se establecía que entraría en vigor cuando lo haga el real decreto que apruebe los estatutos del Organismo Autónomo ANECA, hecho que se produce a finales del mismo año, con la aprobación del Real Decreto 1112/2015, de 11 de diciembre, por el que se aprueba el Estatuto del Organismo Autónomo Agencia Nacional de Evaluación de la Calidad y Acreditación.

\section{La organización en el nuevo sistema: las comisiones de acreditación}

Un aspecto fundamental en el nuevo sistema es la organización de la evaluación, en la que tiene un papel central ANECA. Pero es en la nueva configuración de las comisiones de acreditación donde encontramos novedades más relevantes, especialmente tanto en su estructura y composición como en la designación de sus miembros.

\subsection{ANECA como instrumento de evaluación y acreditación del profesorado}

ANECA se ha convertido, desde su creación, en la piedra angular del sistema de acreditación. La LOMLOU autorizó su creación, de acuerdo con la Ley de Agencias Estatales para la mejora de los servicios públicos, y con la función de "elevar informes al ministerio competente en materia de universidades y al Consejo de Universidades sobre el desarrollo de los procesos de evaluación, certificación y acreditación en España".

Dicha creación se produjo por Acuerdo del Consejo de Ministros, de 19 de julio de 2002, y a través del Real Decreto 1052/2002, de 11 de octubre ${ }^{3}$, comenzando a evaluar a los candidatos a obtener una plaza de profesor universitario. A pesar de su denominación, ANECA no era formalmente una agencia, sino una fundación, lo que limitaba su capacidad de toma de decisiones. Las agencias se regían en el momento de la creación de ANECA por la Ley 28/2006, de 18 de julio, de Agencias estatales para la mejora de los servicios públicos. En cambio, las fundaciones públicas lo hacían por la Ley 50/2002, de 26 de diciembre, de fundaciones. Ello suponía que ANECA no podía ejercer potestades públicas, sino sólo asumir actividades relacionadas con las competencias de las entidades del sector público estatal fundadoras sin que ello significara que se asumieran como competencias propias (art. 46.1 b). Como consecuencia, la certificación de la acreditación se atribuye al Consejo de Universidades (art. 28 LOU y RD 1677/2009, de 13 de noviembre, que aprueba el Consejo de Universidades).

A través de la Ley 15/2014, de 16 de septiembre, de racionalización del Sector Público y otras medidas de reforma administrativa, se concentra en único organismo tanto la propia ANECA como la Comisión Nacional Evaluadora de la Actividad Investigadora

\footnotetext{
${ }^{3}$ Real Decreto 1052/2002, de 11 de octubre, por el que se regula el procedimiento para la obtención de la evaluación de la Agencia Nacional de Evaluación de la Calidad y Acreditación, y de su certificación, a los efectos de contratación de personal docente e investigador universitario.
} 
(CNEAI), que tiene como una de sus funciones más relevantes la evaluación de la labor investigadora a través de los sexenios de investigación. En su art. 8, crea la Agencia Nacional de Evaluación de la Calidad y Acreditación (ANECA), como organismo autónomo de los previstos en el artículo 43.1.a) LOFAGE, con las funciones contenidas en el art. 32 LOU -nuevamente modificado-, y adscrito al Ministerio de Educación, Cultura y Deporte a través de la Secretaría General de Universidades. Finalmente, se transforma en organismo autónomo a través de la aprobación del Real Decreto 1112/2015, de 11 de diciembre, que como ya hemos avanzado supone el inicio del nuevo sistema de acreditación.

No nos detendremos en un análisis de ANECA, aunque sí ponemos de manifiesto el papel fundamental que tiene en el procedimiento de acreditación del profesorado entre otros-, no sólo el funcionario. Su organización y funcionamiento no ha estado nunca exento de opiniones enfrentadas, con razones como la excesiva burocratización, la falta de transparencia o los criterios utilizados en las evaluaciones, entre otros ${ }^{4}$. En cualquier caso, como veremos, ANECA se refuerza con el nuevo sistema, especialmente en lo que se refiere a las comisiones de acreditación, y la figura del Director.

\subsection{La organización de las nuevas comisiones de acreditación}

Uno de los aspectos que presenta más cambios es el referente a la organización de las nuevas comisiones de acreditación, de los que destacamos tres cambios, sobre su constitución y modificación, su estructura y composición, y, por último, el sistema de elección de sus miembros.

\section{a) Constitución y modificación de las comisiones}

La reforma introduce un nuevo redactado en el art. 4 del RD 1312/2007. Se sustituye la antigua redacción de su apartado segundo que establecía que el número de comisiones podía ser revisado por el Ministerio de Educación y Ciencia, a propuesta del Consejo de Universidades o de ANECA. Con la reforma, las Comisiones pasan a depender administrativamente del departamento de ANECA competente para la evaluación del profesorado "y disfrutarán de autonomía para el ejercicio de sus funciones." A diferencia del sistema anterior, el Director de ANECA puede proponer, cada dos años, el incremento o la reducción del número de comisiones, y la distribución de áreas de conocimiento en cada una.

Como ya hemos indicado, ANECA retiene para sí algunas facultades anteriormente asignadas a otras instancias administrativas. En este caso, el Consejo de Universidades deja de tener la facultad de proponer cambios, y la propia configuración de las comisiones pasa a depender enteramente de ANECA, a propuesta de su Director.

Pero el principal cambio en lo que se refiere a las comisiones de acreditación en el nuevo sistema es que dejan de haber 11 (cinco para el cuerpo de TU y cinco para el de CU, además de una para la DA 1a y DA 3a del Real Decreto 1312/2007), para ampliarse

\footnotetext{
${ }^{4}$ Una prueba de ello es la diversidad de opiniones entre los expertos, a menudo críticas, sobre ANECA (CAMPO URBANO et al., 2013; FERNÁNDEZ SARASOLA, 2015, p. 14 y ss).
} 
a 21, constituidas por agrupación de áreas de conocimiento afines. La justificación es que las comisiones sean más cercanas a cada uno de los ámbitos científico y académico de los solicitantes, y así se garantice el principio de especialización y la calidad de la evaluación ${ }^{5}$. Se ha defendido que es razonable esta mayor especialización por cuanto el hecho que las comisiones se refieran a grandes áreas puede comportar una mayor dificultad para evaluar correctamente al candidato (AGUADO, 2014, 8), por lo que las comisiones con la nueva configuración deberían contar con un conocimiento más experto para evaluar las propuestas de los candidatos.

\section{b) Estructura y composición de las comisiones}

La composición de cada una de las comisiones será variable -entre 7 y 13 miembros $^{-6}$, y no se modifican el perfil y los requisitos que deben cumplir sus miembros. Uno de los miembros será el presidente, otro secretario y el resto vocales.

Sí se introduce, a nivel organizativo, una modificación relevante, como es la supresión de los tres tipos de comisiones (para la acreditación de TU, CU, y DA 1a y $3^{a}$ del Real Decreto 1312/2007), como ya hemos visto, y su sustitución por una modificación de la estructura interna de las propias comisiones. Con el nuevo sistema, se crea una subcomisión de acreditación para el cuerpo de $\mathrm{CU}$, integrada exclusivamente por CU miembros de la comisión de acreditación -o profesores de investigación y expertos de reconocido prestigio internacional de categoría equivalente-. Dichos miembros tienen la competencia exclusiva en la evaluación y resolución de las solicitudes de acreditación para el cuerpo de $\mathrm{CU} .{ }^{\prime 7}$

La nueva estructuración de las comisiones conlleva una serie de consecuencias, algunas de ellas establecidas de forma expresa, otras que se producen de forma indirecta. La primera de ellas es que los miembros TU sólo podrán participar en la evaluación de candidatos al cuerpo de TU, mientras que los miembros CU podrán hacerlo en cualquiera de las candidaturas presentadas. Ello hace que se establezcan dos tipos de miembros, en función de su cuerpo de pertenencia. En el sistema anterior podía haber miembros TU y CU en las comisiones para la acreditación para el cuerpo de TU, y sólo CU para el cuerpo de CU, pero la limitación estaba definida en el acceso a la comisión, y en la competencia de cada uno de los dos tipos de comisión (TU o CU). En el nuevo sistema, la limitación es diferente en función de la categoría del miembro.

\footnotetext{
5 Según se dice en la exposición de motivos del RD 415/2015, "Se pretende establecer comisiones mucho más cercanas al ámbito científico y académico de los solicitantes, siguiendo en este punto la práctica que ya existe en países de nuestro entorno, como Francia e Italia. Con este tipo de comisiones se garantiza el principio de especialización en la evaluación del profesorado, así como la calidad del sistema de evaluación."

${ }^{6}$ En el sistema vigente hasta 2015, había un mínimo de 7 pero no un máximo. En la última composición del sistema anterior, el número de miembros de las Comisiones de Acreditación estaba entre 8 y 12: Artes y Humanidades (CU: 9; TU: 8), Ciencias (CU: 10; TU: 10), Ciencias de la Salud (CU: 9; TU: 9), Ingeniería y Arquitectura (CU: 10; TU: 10), Ciencias Sociales y Jurídicas (CU: 10; TU: 12), Disposiciones adicionales primera y tercera del Real Decreto 1312/2007 de 5 de octubre (10). El 23 de febrero de 2016 se nombraron las primeras comisiones, todas ellas con 7 o 9 miembros.

${ }^{7}$ En el mismo sentido, vid. la nueva redacción del art. 15.3 RD 1312/2007
} 
Un segundo cambio que se deriva de esta nueva estructura es el que se produce respecto a la posición mayoritaria de los $\mathrm{CU}$ en las comisiones que valoren las solicitudes de acreditación para el cuerpo de TU. Hasta ahora podían formar parte de las comisiones los CU, los TU y el "personal investigador perteneciente a centros públicos de investigación o por expertos de reconocido prestigio internacional." Con la nueva redacción, dichas comisiones deben estar compuestas de forma mayoritaria por CU, además de por profesores TU y-de forma potestativa- por personal investigador y expertos.

Finalmente, se explicita que dos tercios del total de miembros de cada Comisión deben ser CU -o investigadores y expertos con categoría equivalente- y el tercio restante TU o investigadores con categoría equivalente- ${ }^{8}$, con lo que se refuerza la posición dominante del cuerpo de CU en las comisiones de acreditación.

\section{c) Sistema de elección de los miembros}

El sistema de elección de los miembros de las comisiones de acreditación presenta algunos cambios relevantes. El primero de ellos es que la elección como miembros corresponde al Director de ANECA -antes lo era ANECA de forma genérica- que pasa a tener la iniciativa de proponer tres candidatos -antes eran cinco- al Consejo de Universidades, y un número de suplentes equivalente a la mitad -antes era el mismo número de suplentes que de miembros-. Vemos otro ejemplo del aumento de funciones del Director de ANECA, y una mayor capacidad para proponer candidatos, en una terna que queda reducida a sólo tres candidatos.

Por otra parte, el período de vigencia de los nombramientos sigue siendo de dos años, pero se establece la posibilidad de renovar el nombramiento para dos años adicionales, a propuesta de ANECA, que puede prolongar nombramientos en las comisiones de forma que éstos alcancen un período de cuatro años, el doble que con el sistema anterior.

Otro cambio relevante en lo que se refiere a la elección de los miembros de las comisiones lo encontramos en el mecanismo utilizado. En el sistema anterior, los miembros eran propuestos por ANECA, en función de la experiencia docente e investigadora y de evaluación de los candidatos. Se procuraba, además, la diversidad de disciplinas, de territorios y de género -criterios que se mantienen-. En el nuevo sistema, ANECA realiza un sorteo ${ }^{9}$ entre el personal funcionario en activo de los cuerpos docentes universitarios que reúna los siguientes requisitos:

- Los CU y personal investigador equivalente deberán haber obtenido al menos tres sexenios de investigación, y los profesores TU y personal investigador con categoría equivalente deberán estar en posesión de al menos dos de ellos.

\footnotetext{
${ }^{8}$ En las primeras comisiones, nombradas el 23 de febrero de 2016, la composición ha sido la siguiente: Comisiones de 9 miembros: $6 \mathrm{CU}, 3$ TU; Comisiones de 7 miembros: 5 CU, 2 TU

${ }^{9}$ Sistema que ya existía en el sistema de habilitación, y que había sido propuesto por el Consejo de Universidades (Medidas de mejora del proceso de acreditación nacional para el acceso a los cuerpos docentes universitarios, 20 de julio de 2009), y por algunos autores (DELGADO, F.J. y FERNÁNDEZ-LLERA, R., 2012).
} 
- El último periodo reconocido deberá haberlo sido en los últimos 10 años.

- Deberán tener una antigüedad en sus respectivos cuerpos de al menos 2 años.

- Al menos dos tercios de los miembros de la comisión deberán contar con una experiencia docente universitaria no inferior a 10 años.

En cuanto a la presidencia de las comisiones, se cambia el criterio por el cual ésta se atribuía al miembro de mayor rango académico y antigüedad entre los funcionarios públicos. En el nuevo sistema, el Director de ANECA nombra a los miembros seleccionados por el Consejo de Universidades, y designa -y nombra- a los presidentes y secretarios de cada comisión, atendiendo a los criterios de antigüedad, experiencia en gestión o evaluación, o méritos científico-técnicos. Todos deben formar parte del cuerpo de CU. La razón esgrimida para ello es que el funcionamiento interno del órgano quede encomendado a "personas con experiencia y habilidades para el ejercicio de dicha función".

A ello debe objetarse, como ya se ha dicho, que se cambian criterios reglados por el establecimiento de una amplia discrecionalidad por parte de ANECA $^{10}$. En concreto el Director de ANECA pasa a tener no sólo las potestades ya indicadas, sino que puede también iniciar y resolver un expediente de separación de un miembro de su comisión en caso de incumplimiento del Código Ético.

\section{Algunas cuestiones sobre el proceso de acreditación}

Mención aparte de los cambios organizativos ya descritos, y los referentes a los méritos alegables, que veremos más adelante, hay tres aspectos que debemos tener en cuenta por su relevancia en el proceso de acreditación. Una primera, la supresión de la acreditación universal, y dos de carácter procedimental, como son la eliminación de los informes externos y los cambios en lo que se refiere a las reclamaciones ante resoluciones desestimatorias.

\subsection{Supresión de la acreditación universal}

Con el sistema anterior, los profesores acreditados lo estaban para concurrir a plazas en cualquier rama y área de conocimiento. En el nuevo sistema, la acreditación obtenida produce efectos en una de las cinco ramas de conocimiento previstas (Artes y Humanidades, Ciencias, Ciencias de la Salud, Ciencias Sociales y Jurídicas, e Ingeniería y Arquitectura), con lo que se elimina la acreditación universal. Como se dice en la exposición de motivos del RD 415/2015, "De este modo se garantiza que cuantos obtengan una acreditación en una rama de conocimiento hayan sido evaluados conforme a criterios semejantes y estándares análogos, que no necesariamente serán los mismos en otras ramas."

\footnotetext{
${ }^{10}$ Como bien indica Aguado Cudolà, dicho cambio “(...) conllevaría substituir los criterios reglados mencionados por el establecimiento de una amplia discrecionalidad administrativa. Tal solución resulta discutible por cuanto, a mi juicio, parecería preferible evitar que se produjera un eventual control de los presidentes por los órganos designantes en base a criterios amplios." (AGUADO, 2014, 8).
} 
Las comisiones ya tenían dicha disposición en el sistema anterior. Por tanto, la comisión de Ciencias Sociales y Jurídicas podía acreditar a un profesor como TU, y que dicha acreditación tuviera carácter universal, es decir, que dicho TU pudiera acabar optando por una plaza en Artes y Humanidades, por ejemplo. Por otro lado, con el nuevo sistema, aproximamos más el foco, pero mantenemos el esquema. La comisión de Derecho puede acreditar a un profesor como TU, y dicha acreditación tiene efectos en el área de Ciencias Sociales y Jurídicas, es decir, que dicho TU puede acabar optando por una plaza en Economía o Sociología.

Se quiere preservar, en cualquier caso la posibilidad de valorar la multidisciplinariedad. En la nueva redacción del art. 3 que introduce el RD 415/2015 se añade que "Se dispondrán, en todo caso, procedimientos para que los solicitantes que desarrollen una especialización de carácter multidisciplinar o en ámbitos científicos interdisciplinares en los que concurran dos o más ramas diferentes puedan, como resultado de un mismo proceso de evaluación, ser acreditados en más de una rama." En consecuencia en el art. 13.1 se establece que se optará a la acreditación "por una o varias ramas del conocimiento".

\subsection{Eliminación de los informes externos y reforzamiento de la capacidad de las co-}

\section{misiones}

Un cambio fundamental en el nuevo sistema es que las decisiones se adoptan de forma colegiada, a partir de dos ponencias elaboradas por dos miembros de la Comisión. Solo en casos de discrepancias entre los ponentes que la Comisión no se considere en condiciones de solventar el Presidente podrá, con carácter excepcional, solicitar un informe a un experto externo perteneciente al área de conocimiento del solicitante. Ello supone en la práctica suprimir los informes externos. Se mueve por tanto el centro de decisión de las acreditaciones de los evaluadores externos a dos miembros de la comisión, que -es de suponer- preferentemente serán los pertenecientes a la disciplina del candidato.

Efectivamente, la eliminación de la remisión de las solicitudes a dos expertos (se elimina el contenido del art. 15.2), se trata de un cambio sustancial. En el nuevo sistema, según se establece en el nuevo redactado del art. 15.4, “(...) cada solicitud será informada por, al menos, dos miembros de la Comisión, que actuarán como ponentes. La Comisión adoptará la decisión colegiadamente, a la vista de la documentación presentada y de los informes de los ponentes. En casos de discrepancia entre los ponentes que la Comisión no se considere en condiciones de solventar, el Presidente podrá, con carácter excepcional, solicitar un informe a un experto externo perteneciente al área de conocimiento del solicitante, cuyo informe no tendrá carácter vinculante. Se valorarán preferentemente la actividad investigadora y la actividad docente. La formación académica, la transferencia de conocimiento y actividad profesional, así como la actividad de gestión serán valoradas únicamente para compensar insuficiencias no graves en alguna de las dos dimensiones objeto de valoración preferente."

A pesar de que en el sistema anterior, la decisión formal se tomaba en el seno de las comisiones, los informes externos eran un elemento de juicio importante en la forma- 
ción de la decisión de la comisión en cada caso concreto. El papel "experto" de los informes externos ha sido puesto en valor por la jurisprudencia, afirmando que "el principio de competencia científica queda plenamente salvaguardado mediante la previsión de los informes de los expertos", recogida en el art. 15 RD 1312/2007 ${ }^{11}$.

En el nuevo sistema, los informes de los expertos se sitúan en un segundo plano, ya que pasan a ser excepcionales y no vinculantes. La utilización de dichos informes había sido criticada en cuanto que los expertos podían ser profesores ajenos a la disciplina, y por tanto carecer de conocimiento específico en un área concreta ${ }^{12}$.

\subsection{El procedimiento de revisión de las resoluciones de acreditación}

El nuevo sistema de acreditación no altera el sentido del silencio administrativo, aunque se cambia la redacción del art. 15.6 RD 1312/2007, explicitando su carácter desestimatorio. Por otra parte, se mantiene la imposibilidad de presentar una nueva solicitud hasta transcurridos 18 meses desde la presentación de la solicitud evaluada desfavorablemente.

También se mantiene, como en el sistema anterior, la posibilidad de presentar una reclamación contra las resoluciones de las comisiones de acreditación, en el plazo de un mes, ante el Consejo de Universidades que, en el caso de ser admitida a trámite, será valorada por una Comisión designada al efecto por dicho órgano. Dicha Comisión de Reclamaciones del Consejo de Universidades también se mantiene, y valorará las reclamaciones que se presenten contra las resoluciones de las Comisiones de Acreditación, ratificando las resoluciones o aceptando las reclamaciones. En éste último caso, la resolución de la Comisión -que debe incluir los aspectos a revisar- se remite a ANE$\mathrm{CA}$, donde se crean cinco Comisiones de Revisión ${ }^{13}$, una por cada una de las ramas de conocimiento (Artes y Humanidades, Ciencias, Ciencias de la Salud, Ciencias Sociales y Jurídicas, e Ingeniería y Arquitectura), que tendrán las siguientes características:

\footnotetext{
${ }^{11}$ Según se afirma en el FJ 3o de la Sentencia de la Audiencia Nacional, de 11 de septiembre de 2011, "el conocimiento de los expertos puede tener relevancia a los efectos de que el interesado pueda cuestionar su propia condición de tales o, en su caso, recusar a alguno de ellos. Ha de notarse, sin embargo, que la decisión correspondiente es, formal y materialmente, de la Comisión de Acreditación de la rama de conocimiento de la que se trate, y no de los expertos, cuya labor es de puro asesoramiento." (FJ 3으 SAN 3675/2013). En el mismo sentido, FJ 5ㅇ de la Sentencia de la Audiencia Nacional, de 10 de diciembre de 2015 (SAN 4391/2015)

12 En este sentido, "La presencia de miembros de otros ámbitos diferentes al del candidato, algo que, de nuevo, en teoría puede resultar positivo para introducir elementos de comparabilidad externa y evitar endogamias "científicas», también ha sido considerado como un elemento de distorsión, ya que la comisión de resolución quedaba, en buena medida, en manos de los informes de los expertos... algunos de los cuales no lo era propiamente ya que no pertenecía a la misma área de conocimiento que el postulante a la acreditación." (ANDRADAS, C.; GONZÁLEZ, J., 2012, 207). En un sentido similar, DEL VALLE PASCUAL $(2008,1730)$.

13 Según la exposición de motivos del RD 415/2015 “(...) permitirán un funcionamiento más eficiente de esta fase del procedimiento y reforzarán las garantías de los interesados en la reclamación contra resoluciones negativas, reduciendo previsiblemente la litigiosidad."
} 
- Compuestas cada una de ellas por 7-11 miembros.

- Se procurará que los miembros cuenten con experiencia previa como evaluadores en las comisiones de acreditación.

- Dependerán administrativamente del órgano de ANECA competente para la evaluación del profesorado.

- Dispondrán de autonomía para el ejercicio de sus funciones.

- Integradas exclusivamente por $\mathrm{CU}^{14}$.

- Los miembros deben reunir los mismos requisitos que para serlo de una comisión de acreditación (en el caso de los CU -o equivalentes-, tres sexenios de investigación).

- Los miembros son seleccionados por el Consejo de Universidades a propuesta de ANECA - incluidos el Presidente y el Secretario-, y nombrados por el Director de ANECA a propuesta del Consejo de Universidades.

- Nombramiento por dos años, prorrogables por otros dos.

Antes de emitir su evaluación, las comisiones de revisión podrán recabar el informe no vinculante- de un experto externo perteneciente al área de conocimiento del solicitante. El informe de evaluación de la comisión de revisión es vinculante, y se remite al Consejo de Universidades para que dicte una resolución, que pone fin a la vía administrativa, por lo que sólo cabe el recurso de reposición o la vía contenciosoadministrativa $^{15}$.

\section{La valoración de los méritos en el nuevo sistema}

La evaluación de la investigación es un tema controvertido, y como hemos visto muchas han sido las críticas a los diferentes sistemas implantados, aunque todos ellos han aportado un intento de racionalizar y hacer más objetivo el proceso evaluador. No es lugar éste para abordar este debate, sino que nos centraremos en las novedades que el nuevo sistema de acreditación presenta en lo que se refiere a la evaluación en sí de los méritos de los candidatos. Lo haremos muy especialmente en tres aspectos, como son la supresión de la acreditación universal, los méritos de investigación y docencia y, por último, su baremación.

\subsection{Discrecionalidad técnica y criterios de valoración de los méritos en el proceso de evaluación}

Si existe un concepto especialmente relacionado con la evaluación de la investigación en el caso que nos ocupa ese es el de la discrecionalidad técnica. Las comisiones tienen un margen elevado de discrecionalidad, más si cabe al resultar prácticamente eliminados los informes externos. Ello hace que el peso de la decisión recaiga en los miembros

\footnotetext{
14 “También podrá formar parte de ellas el personal investigador perteneciente a centros públicos de investigación de categoría equivalente a la de catedrático de universidad, así como expertos de reconocido prestigio internacional." (art. 16.6 RD 1312/2007)

${ }^{15}$ En la anterior redacción del art. 16.5 la resolución de la Comisión de reclamaciones podía ser recurrida en alzada ante la presidencia del Consejo. En el nuevo redactado se suprime dicha posibilidad, y se establece, en el nuevo art. 16.9, que "La resolución del Consejo de Universidades pondrá fin a la vía administrativa y frente a la misma podrá interponerse recurso de reposición o bien ser recurrida directamente ante la jurisdicción contencioso-administrativa."
} 
de las comisiones de acreditación, y en especial en los ponentes de los informes sobre los que debe basarse la resolución de acreditación. Hay que añadir que la jurisprudencia ha insistido de forma reiterada en que su contenido no está sujeto a control jurisdiccional, en tanto que se trata de una decisión basada en la discrecionalidad técnica de la comisión de acreditación ${ }^{16}$, lo que no quiere decir que la actividad evaluadora de las comisiones esté fuera del control de los tribunales, sino que existe un núcleo reservado, sujeto exclusivamente a la valoración técnica de la comisión de acreditación ${ }^{17}$.

Como se ha dicho, existe una "fuerte tensión entre las garantías constitucionales de los candidatos a acreditaciones o plazas y la libertad de evaluadores, administradores e instituciones para evaluar y seleccionar a los candidatos de modo discrecional (...)", afirmando incluso que "uno de los mayores problemas de nuestros sistemas de evaluación del profesorado sea la excesiva discrecionalidad técnica, rayana con la arbitrariedad y sujeta a la apreciación particular e inmotivada de los evaluadores" (GALÁN GONZÁLEZ, GONZÁLEZ GALÁN y RODRÍGUEZ-PATRÓN., 2014, 157). Si bien no podemos estar de acuerdo en esta última afirmación, si existe, desde el punto de vista jurídico, dicha confrontación entre las garantías de los candidatos y la discrecionalidad necesaria para que las comisiones puedan evaluarlos con la mayor objetividad posible.

Ahora bien, dicha discrecionalidad por parte de las comisiones de acreditación se ve limitada por diferentes elementos que intentan aportar objetividad y previsibilidad al proceso. Como se ha dicho, establecer criterios operativos de evaluación es la condición más importante para incrementar la validez y la fiabilidad entre evaluadores en los procesos de selección del profesorado. "Pero, además, el conocimiento de los criterios y del baremo por parte de los candidatos no sólo hace posible un sistema más justo de selección de profesores, sino que también permite perfilar y orientar la carrera docente de aquellos profesores y/o investigadores que algún día pretendan conseguir la condición de profesor funcionario universitario." (BUELA-CASAL, 2007, 474) ${ }^{18}$.

Dichos criterios estaban contenidos, a nivel general, en el RD 1312/2007, y se desarrollaban en el documento de ANECA Principios y orientaciones para la aplicación de los criterios de evaluación ${ }^{19}$, que concretaba los méritos que pueden alegarse para obtener la acreditación, y además, una serie de tablas de puntuación en cada uno de los méritos alegables. El nuevo sistema traslada parte de los requisitos al texto del RD

\footnotetext{
16 Por ejemplo, la AN afirma que "los juicios técnicos de los órganos de selección en las pruebas de ingreso en la Administración (o de evaluación, como es el caso) no son susceptibles de control jurídico por la Jurisdicción, y corresponden en exclusiva a aquellos órganos. En el núcleo de la valoración técnica la jurisdicción, que opera con criterios jurídicos, no se puede subrogar en el lugar de la Comisión o Tribunal evaluador, sin perjuicio de que el uso de la discrecionalidad técnica pueda, y deba ser, objeto de control jurisdiccional desde el exterior de ese núcleo reservado, en función de criterios de carácter jurídico, como son fundamentalmente la interdicción de la arbitrariedad y el principio de igualdad de acceso a los cargos públicos ( artículos 9.3 y 23.2 C.E .), y sobre la base de datos fácticos o jurídicos diferentes en todo caso de la pura valoración de los méritos en su dimensión técnica" SAN 4391/2015, de 10 de diciembre de 2015.

17 Como ha afirmado el TC de forma reiterada, en el caso de los sexenios de investigación, "lo que no pueden hacer los Tribunales de Justicia es sustituir en las valoraciones técnicas a los órganos administrativos calificadores" FJ 50 STC 17/2009, de 26 de enero de 2009.

${ }^{18}$ En un sentido similar, ANDRADAS y GONZÁLEZ $(2012,206)$.

${ }^{19}$ Principios y orientaciones para la aplicación de los criterios de evaluación, ANECA, 2012
} 
1312/2007, en forma de méritos obligatorios de investigación y docencia, y establece, también el mismo texto, un baremo para la obtención de la acreditación, que flexibiliza los requisitos mínimos en determinadas situaciones.

\subsection{Los méritos de investigación ${ }^{20}$}

Encontramos un cambio relevante en los méritos obligatorios que un candidato debe cumplir, que aparecen reflejados en el texto del RD. No hay, por otra parte, cambios relevantes respecto al tipo de méritos que pueden alegarse ${ }^{21}$. Vemos ambos apartados a continuación.

\section{a) Los méritos obligatorios de investigación y docencia}

El principal cambio por lo que se refiere a los méritos necesarios para conseguir una evaluación positiva, es la introducción de méritos obligatorios, requisitos que de forma parcial constaban anteriormente en los criterios de evaluación de ANECA, y que ahora -en parte- se reflejan en el texto del RD 415/2015. Este cambio da valor normativo a una serie de méritos de carácter obligatorio, desplazando su contenido de un documento de criterios aprobado por ANECA a la principal norma que desarrolla el procedimiento de acreditación.

Entre los méritos de investigación de carácter obligatorio encontramos la necesidad de justificar un número mínimo de contribuciones científicas (artículos, libros, capítulos de libro o resultados de producción científica, técnica o artística publicados), que propondrá cada comisión, "teniendo en cuenta, cuando resulte procedente, los estándares e índices bibliométricos nacionales e internacionales acreditados." El propio RD establece un mínimo, cuando dispone que los candidatos presentarán las cuatro contribuciones más relevantes en su trayectoria científica, para que se evalúe su calidad e impacto en su área de especialización.

En el caso concreto de la acreditación para el cuerpo de CU, se establece como requisito obligatorio "(...) que los solicitantes aporten indicios significativos de una trayectoria de liderazgo y reconocimiento externo a la institución donde prestan servicios, tanto en lo relacionado con la actividad docente como con la investigadora."

Respecto a la docencia, se exigirá un número de años de experiencia docente y una valoración positiva de la misma, aunque se excluye de esta obligación a los solicitantes que hayan desarrollado su carrera académica en una institución no universitaria o no española -bajo ciertas circunstancias-, siempre y cuando acrediten resultados de investigación excepcionales.

\footnotetext{
${ }^{20}$ Hacemos notar un cambio, que puede parecer puramente semántico, como es el de cambiar la denominación de criterios por méritos. El foco se ponía en el anterior sistema en los criterios que las comisiones y los evaluadores debían seguir, mientras que en el nuevo lo hace en los méritos, que el candidato debe certificar.

${ }^{21}$ Para un análisis de los cambios en los criterios de evaluación, vid. ORTIZ DE URBINA CRIADO y MORA VALENTÍN (2013)
} 
Como vemos, se añaden unos requisitos obligatorios pero al mismo tiempo se hace una remisión a los criterios mínimos que establezca cada comisión, que dada la mayor especialización de las nuevas comisiones de acreditación pueden ser más concretos. Por lo tanto, son méritos obligatorios pero sujetos a un elevado grado de indeterminación, que debe ser concretado por las comisiones.

\section{b) La clasificación de los méritos}

No se produce por otra parte un cambio relevante sobre los méritos a alegar en el proceso de acreditación ${ }^{22}$, aunque sí una reorganización de los apartados, con la introducción de un nuevo apartado de transferencia de conocimiento y experiencia profesional, que tendrá una mayor importancia en determinadas áreas. Se matizan algunos criterios -por ejemplo, que los proyectos o contratos deben tener resultados constatables-, y se eliminan la mayor parte de los incisos finales que -en forma de "etcétera" o "etc."-, dejaban la puerta abierta a la interpretación de los criterios.

i) Actividad investigadora: El cambio fundamental en los criterios de evaluación en lo referente a la actividad investigadora es consecuencia directa de la creación de un nuevo apartado sobre transferencia de conocimiento, hacia el cual se trasladan algunos méritos alegables, mientras que los cambios de contenido son menores.

ii) Actividad docente: Al igual que en la evaluación de la actividad investigadora, también en el apartado de actividad docente desaparecen las referencias a la actividad profesional, para ser trasladadas al nuevo apartado de transferencia, empezando por el propio título, que pierde la denominación de "profesional". De la misma forma, los cambios en referencia a los méritos a alegar son menores, y se limitan a eliminar algunas imprecisiones.

iii) Formación académica: En cuanto a la formación académica, se mantienen los dos apartados de formación -predoctoral y posdoctoral-, aunque todo lo relativo a la tesis doctoral pasa al apartado predoctoral. El cambio más relevante es que todo lo relativo a la tesis doctoral ("premio extraordinario de doctorado y otros premios, mención de doctorado europeo o internacional, mención de calidad o excelencia del programa de doctorado"), que anteriormente estaba en el epígrafe posdoctoral, pasa a estar en el ahora denominado en consecuencia "predoctoral y doctoral", mientras que los méritos posdoctorales se restringen por tanto a las becas o contratos exclusivamente posdoctorales.

iv) Transferencia de conocimiento y experiencia profesional: Este nuevo apartado se nutre básicamente de los anteriores apartados 1. C (Calidad de la transferencia de los resultados) y 2. D (Calidad y dedicación a actividades profesionales), a los que se añaden nuevos elementos. Como ya se ha indicado, la propia exposición de motivos del RD 415/2015 destacaba que la inclusión de la valoración de la transferencia y la actividad profesional de forma autónoma era una novedad muy importante para determinados ámbitos científicos. La creación de este apartado pretende evitar la infravaloración de los méritos de transferencia y actividad profesional en determinados

\footnotetext{
${ }^{22}$ Sobre los cambios en los méritos, vid. GALINDO CALDÉS y ARGUIMBAU VIVÓ (2016, 12 y ss.)
} 
ámbitos, por lo que es de prever que será relevante para la evaluación en determinadas comisiones evaluadoras.

v) Experiencia en gestión y administración educativa, científica y tecnológica: Prácticamente no hay novedades respecto a los méritos sobre experiencia en gestión y administración educativa, científica y tecnológica, más allá de que se suprime el mínimo de un año en un cargo o puesto para ser valorado. También se acota la expresión última de "otros méritos" a la gestión.

\subsection{El cambio en los baremos en las diferentes dimensiones de la acreditación}

No ha habido, como hemos visto, un cambio relevante en cuanto a los méritos que pueden alegarse en la acreditación. No podemos decir lo mismo del sistema de baremación, que sufre un cambio muy relevante.

A pesar de que se enfatiza la priorización de las dos dimensiones "esenciales", como son la investigación y la docencia ${ }^{23}$, se consideran diferentes opciones en las que una calificación insuficiente puede compensarse con méritos relevantes en transferencia de conocimiento y experiencia profesional, en gestión o -en el caso de la acreditación para profesores TU-, formació ${ }^{24}$. Teniendo en cuenta que con el antiguo sistema ambas conjuntamente suponían un $90 \%$ de la puntuación, vemos que las restantes dimensiones cobran protagonismo.

La nueva baremación sustituye la puntuación numérica (0-100) por una calificación alfabética (excepcional (A), bueno (B), compensable (C), insuficiente (D) y circunstancia especial (E)), aunque no se concreta qué se entiende por cada una de las calificaciones, por lo que se deja un margen importante a la discrecionalidad. Según la exposición de motivos, "Corresponderá a ANECA la aprobación de los criterios de evaluación de cada uno de los cuerpos docentes universitarios y de los distintos campos, ajustándolos para mayor consistencia del modelo global, si bien cada Comisión propondrá los que estime apropiados en cada campo. De forma análoga a lo que ha venido sucediendo con las convocatorias anuales de la CNEAI, los criterios para la acreditación en cada ámbito científico se publicarán y se revisarán periódicamente cada dos años."

\footnotetext{
${ }^{23}$ En el nuevo art. 15.3 se afirma que "Se valorarán preferentemente la actividad investigadora y la actividad docente. La formación académica, la transferencia de conocimiento y actividad profesional, así como la actividad de gestión serán valoradas únicamente para compensar insuficiencias no graves en alguna de las dos dimensiones objeto de valoración preferente."

${ }^{24}$ Como se afirma en la exposición de motivos del RD 415/2015, “(...) una evaluación positiva de estas dos dimensiones conforme a lo dispuesto en el presente real decreto hará innecesario tener en cuenta más méritos para conseguir la acreditación. En el caso de que un solicitante no alcance el nivel mínimo exigible, y siempre que la insuficiencia no sea grave, ésta se podrá compensar en virtud de méritos relevantes en las tres dimensiones restantes: transferencia de conocimiento y actividad profesional; gestión; y formación, esta última evaluable solo a quienes soliciten la acreditación para el cuerpo de profesores titulares de universidad."
} 
Baremación para la acreditación para el cuerpo de profesores CU y TU (calificación mínima)

\begin{tabular}{|c|c|c|c|c|c|}
\hline & Investigación & Docencia & $\begin{array}{l}\text { Transf./Act. } \\
\text { prof. }\end{array}$ & Gestión & Formación \\
\hline \multirow{6}{*}{$\mathrm{CU}$} & $B$ & $B$ & & & - \\
\hline & $A$ & $C, E^{(1)}$ & & & - \\
\hline & $B$ & $C$ & B & & - \\
\hline & B & $C$ & & $B$ & - \\
\hline & C & $B$ & $A$ & & - \\
\hline & $C$ & B & & $A$ & - \\
\hline \multirow{5}{*}{ TU } & B & B & & & \\
\hline & A & $C, E^{(1)}$ & & & $\mathrm{B}$ \\
\hline & B & C & B & & B \\
\hline & $B$ & $\mathrm{C}$ & & $B$ & B \\
\hline & C & B & $A$ & & B \\
\hline
\end{tabular}

(1) Solo es válido para la acreditación cuando un solicitante haya desarrollado su carrera principalmente en una institución no universitaria o en una universidad no española donde el cómputo y los instrumentos de medición de la calidad de la actividad docente resulten difíciles de trasladar al sistema español.

EI RD establece una serie de supuestos, o combinaciones de calificaciones, cuya obtención conduce a la acreditación para el cuerpo de CU o profesores TU. La primera de ellas, que reproducimos en la siguiente tabla, describe las diferentes combinaciones que conllevan la acreditación. En todo caso, la formación académica no se tiene en consideración para la acreditación para el cuerpo de CU.

Tanto para la acreditación para CU como para TU nos encontramos con cuatro combinaciones posibles:

1. Haber obtenido una calificación buena (B) en investigación, así como en docencia. Se trataría de la situación "estándar" en una evaluación.

2. Una investigación considerada excepcional (A) y docencia compensable (C) o encontrarse en una circunstancia especial $(E)$.

3. Haber obtenido una calificación buena (B) en investigación, compensable (C) en docencia, y buena (B) en transferencia de conocimiento y experiencia profesional o en gestión.

4. Haber obtenido una calificación buena (B) en docencia, compensable (C) en investigación, y excepcional (A) en transferencia de conocimiento y experiencia profesional (En el caso de las acreditaciones a CU alternativamente excepcional (A) en gestión.

En todos los supuestos, excepto en el segundo, es necesaria la obtención de una calificación buena (B) en formación en el caso de las acreditaciones a TU.

Como puede observarse, el nuevo baremo permite situaciones "estándar", como la primera, pero sobretodo de carácter especial, que pueden compensar generalmente el déficit en méritos considerados esenciales, como son la investigación y la docencia. Ello permitiría acreditar candidatos con gran experiencia investigadora, de transferencia o de gestión -éste último sólo en el caso de las acreditaciones para CU-, que en el anterior sistema no podían superar la evaluación al tener la investigación y la docencia un $90 \%$ de los puntos. 
En conclusión, el nuevo baremo puede ampliar la casuística los candidatos acreditados, aunque deja un margen más amplio para el criterio de las comisiones, y limita la capacidad de los candidatos para rebatir las resoluciones desfavorables.

\section{Conclusiones}

La reforma del sistema de acreditación para el acceso a los cuerpos docentes universitarios aborda temas siempre controvertidos, como la evaluación de la investigación y la selección del profesorado. Aun así, es necesario el establecimiento de mecanismos que garanticen que los candidatos alcancen unos mínimos de calidad, tanto en investigación como en docencia. La transformación de la universidad en los últimos tiempos aconseja también considerar otros aspectos, especialmente la transferencia de conocimiento. Transcurridos casi diez años desde la implantación del sistema de acreditación, se produce una reforma que pretende la simplificación y la mejora regulatoria de los procedimientos de acreditación, así como garantizar mayor objetividad y transparencia en la acreditación, aspectos muy criticados en el anterior sistema.

Para lograrlo, se producen algunos cambios relevantes. Por un lado, se refuerza el papel del cuerpo de CU en el proceso, en tanto que su presencia es mayoritaria en las nuevas comisiones, ocupan su presidencia, y conforman en exclusiva las subcomisiones para la acreditación para CU y las comisiones de revisión. Se refuerza también el papel de ANECA, especialmente la figura del Director, que pasa a disponer de mayores facultades. La reforma también establece unas comisiones más afines a la disciplina, lo que supone un conocimiento más experto sobre los méritos de los candidatos, pero también los riesgos que conlleva la proximidad entre evaluadores y evaluados. La práctica eliminación de los informes externos refuerza esta línea, suprimiendo la evaluación externa. El centro de la discusión sobre el sistema, en definitiva, seguirá gravitando sobre el juicio técnico sobre el que se basa la resolución de la acreditación, antes más centrados en los expertos externos, y ahora en los miembros de las comisiones. Cambia por tanto quién decide efectivamente sobre dicho juicio, pero no tanto el hecho de evaluar en función de unos criterios preestablecidos.

Por otro lado, con la reforma se pretende también flexibilizar el sistema de acreditación, facilitando la incorporación a los cuerpos docentes universitarios de perfiles que no responden a un modelo clásico de carrera académica, pero que disponen de una excelente trayectoria en investigación o transferencia de conocimiento. Se busca aportar mayor objetividad en el proceso, pero dejando cierto margen que permita nuevos perfiles académicos, especialmente investigadores.

\section{Bibliografía}

AGUADO CUDOLÀ, V. (2014). La selección de los cuerpos docentes universitarios: El sistema de acreditación. Revista de Educación y Derecho, 10.

ALEGRE AVILA, J. M. (2007). El nuevo sistema de selección del profesorado univesitario funcionario. Revista española de derecho administrativo, 135, 437-457. 
ANDRADAS HERANZ, C., y GONZÁLEZ GARCÍA, J. V. (2012). Cuestiones prospectivas del profesorado universitario. Revista catalana de dret públic, 44, 191-224.

BASTIDA FREIJEDO, F. J. (2015). La nueva carrera académica: visión senior (con vista cansada). Revista general de derecho constitucional, 21.

BUELA-CASAL, G. (2007). Reflexiones sobre el sistema de acreditación del profesorado funcionario de Universidad en España. Psicothema, 19(3), 473-482.

CAMPO URBANO, S., MUÑOZ RUIZ, E., BERZOSA ALONSO-MARTÍNEZ, C., RUBIO LARA, M. J., COTARELO GARCÍA, R., LAPORTA SAN, M., SOLÉ, C. (2013). La ANECA y la crisis de la Universidad Española. Temas para el debate, 229.

DEL SAZ CORDERO, S. (1997). "Las oposiciones a cátedras", Revista de Administración Pública, 144, 75-95.

DELGADO RIVERO, F. J.; FERNÁNDEZ-LLERA, R. (2012). Sobre la evaluación del profesorado universitario (especial referencia a ciencias económicas y jurídicas). Revista española de documentación científica, 35(2), 361-375.

FERNÁNDEZ SARASOLA, I: Algunas reflexiones en torno a la evaluación de profesorado universitario de ciencias jurídicas, Revista General de Derecho Constitucional, 21, 2015.

GALÁN GONZÁLEZ, A., GONZÁLEZ GALÁN, M. A., RODRÍGUEZ-PATRÓN RODRÍGUEZ, P. (2014). La evaluación del profesorado universitario en España. Sistema nacional y divergencias territoriales. Revista de Educación, 366, 136-164.

GALINDO CALDÉS, R., ARGUIMBAU VIVÓ, L. (2016): El nuevo sistema de acreditación nacional para el acceso a los cuerpos docentes universitarios: novedades del Real Decreto 415/2015, de 29 de mayo, Informes Appendix, 1/2016, Disponible en http://www.appendix.es/informes.

ORTIZ DE URBINA CRIADO, M., MORA VALENTÍN, E. M. (2013). El sistema de acreditación del profesorado a través del Programa ACADEMIA: Evolución y cambios. Revista española de documentación científica, 36(1).

NIETO, A. (1984). La tribu universitaria, Madrid: Tecnos.

VALLE PASCUAL, J. M. DEL. (2008). Primeros apuntes sobre el sistema de acreditación y acceso a la condición de profesor de los cuerpos docentes universitarios. Actualidad administrativa, 15, 1722-1732. 